\title{
An Under Actuated Robotic Arm with Adjustable Stiffness Shape Memory Polymer Joints
}

\author{
Amir Firouzeh ${ }^{1}$, Seyed Sina Mirrazavi Salehian ${ }^{2}$, Aude Billard ${ }^{2}$, Jamie Paik ${ }^{1}$
}

\begin{abstract}
Various robotic applications including surgical instruments, wearable robots and autonomous mobile robots are often constrained with strict design requirements on high degrees of freedom (DoF) and minimal volume and weight. An intuitive design to meet these contradictory requirements is to embed locking mechanism in under actuated robotic manipulators to direct the actuation from a single and remote source to drive different joints on demand. Mechanical clutches do serve such purposes but often are bulky and require auxiliary mechanism making it difficult to justify the high cost adding the additional DoF, especially in $\mathrm{cm}$ scale.

Here, we introduce an under-actuated robotic arm with shape memory polymer (SMP) joints. Through controlling the temperature, the stiffness of the joints can be adjusted and selected joints will be activated while the rest are fixed in their position. The presented prototype can control the joints independently with a coupled actuation from two stepper motors. Since we have redundant DoFs in the arm, there can be more than one configuration to reach a given position. We use a probabilistic technique to determine the optimum configuration with the minimum number of active joints that can yield the desired posture. In this paper, we report on the performance of the proposed design for the hardware and the configuration planner.
\end{abstract}

\section{INTRODUCTION}

As the application of robots expands in demanding fields such as in vivo surgical tools as active catheters and endoscopes [1], [2], exploration robots [3], and dexterous robotic manipulators [4], the need for embedding more degrees of freedom $(\mathrm{DoF})$ while maintaining minimum mass and size is emerging. Cable-driven mechanisms are the prevailing solution to remove the actuators from the joint area [5] in order to miniaturize the manipulator. But in these systems, the overall size and weight of the robot would still be high since the same components are merely placed elsewhere. Moreover, the complexity of fabrication and operation confines number of DoF.

A method to reduce the number of points of actuation while keeping the same work space is to direct the energy from a single point of actuation to a desired set of joints (or a single joint) by locking in place all the rest. Previously, this method was proved to be effective in expanding the work space in an under actuated mechanism that exploits external constraints to selectively fix the joint state in an under actuated arm [8].

\footnotetext{
*This work was supported by Swiss National Center for Competence in Research in robotics.

*This work was supported by EU projects AlterEgo (grant \#600610).

${ }^{1}$ Amir Firouzeh and Jamie paik are with Reconfigurable Robotics Lab at EPFL, Lausanne. Jamie.paik@epfl.ch

${ }^{2}$ Seyed Sina Mirrazavi Salehian and Aude Billard are with Learning Algorithms and Systems Laboratory at EPFL, Lausanne.
}

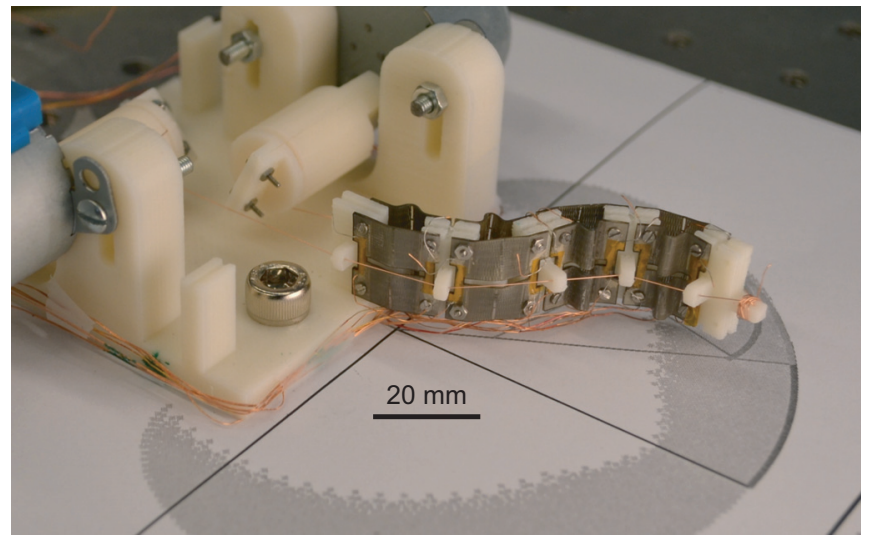

Fig. 1: A four DoF under actuated robotic arm with two stepper motors that actuate different joints through tendons that run along the arm. Each of the four joints can be activated on demand when the embedded heaters in SMP layers are turned on.

Conventional locking mechanisms for directing the actuation to different joints and segments in robotic systems have already been proposed including mechanical locks such as hydraulic cylinders and solenoid valves [9] and mechanical latching [1]. More novel methods such as jamming effect for locking robot's segments has also been studied [10], [11]. Beside the reduced weight and drive simplicity, another advantages of using lock mechanism is the possibility of fixing different joints and segments of the robot in a desired state which increases the load bearing capacity. The main disadvantage of the proposed methods is the complexity of the locking mechanism. For jamming and hydraulic locking, this comes from the auxiliary equipment. Difficulty with tubing in a system with large number of DoF is another challenge in these robots. For mechanical latching, designing and operating the lock mechanism to prevent blocking is the main challenge to make a system with large number of DoF.

In this research, we introduce a locking method for joints based on mechanical property change in robot's body using functional materials. Different methods for controlling stiffness of different segments of the body using phase transition in materials have been proposed including: solid to liquid transition in low melting point metals [12], phase transition in wax [13], and glass to rubbery state transition in polymers [14]. Here, based on ease of fabrication and scalability, we designed a locking mechanism that uses glass transition in polymers. Elasticity modulus of polymers drop orders of magnitude over their glass transition temperature $\left(T_{g}\right)$. By embedding the heaters inside the polymer layer, we can control properties of different segments of a polymeric sheet and 
direct the actuation to the desired joints. Compared to other methods such as mechanical methods and jamming, using this locking mechanism results in a simpler system which is more suitable for miniaturization. Eventually, by controlling the temperature in increments, we would also be able to control the modulus of elasticity of the material and hence joint stiffness continuously instead of having only two states, active or inactive. To have a controlled shape in the rubbery state, we decided to use shape memory polymer (SMP) [15] as the material with adjustable modulus of elasticity. Compared to normal polymers, these are engineered to have better cross-links which enables them to recover the deformation in their rubbery state (high temperature) and return to their memory shape with higher repeatability. Compared to the more common approach where the functional materials are used as actuators in robots to reduce the size and weight [2], [6], [7], in the method studied here, we mainly exploit the mechanical property change in the material.

In systems with redundant DoF, we can have different configurations for reaching a desired tip position. In the last three decades, a variety of algorithms based on numerical [16], [17] or analytical [18] methods have been proposed to use redundancy for minimizing a desired cost function; such as torque or velocity in joints. In our case however, based on the actuation method of the robot (at this point we activate only one joint at a time) the problem is optimizing a function with continuous and discrete parameters. Minimizing the number of joints that need to change their angle for reaching a given position is the optimization problem we will try to solve here. One possible solution is to formulate the problem as a discrete optimization problem. This method requires significant computational power for computing the solution online and moreover the convergence is highly dependent on the initial guess. Instead, this optimization can be treated as a classification problem. By locking a specific joint the workspace of the robot changes. We train a set of probalistic models to represent the distribution of achievable postures (workspace) for each set of active joints. We then determine the minimum number of joints needed by comparing the likelihoods of the presence of a desired position in different models. Then, the inverse kinematic is solved by considering only the active joints to determine the final configuration of the robot.

The main contribution of this work is the introduction of a lock mechanism to direct the central actuation to the desired joint in an under-actuated robotic system. The proposed active locking mechanism works based on the principle of adjusting the stiffness of an SMP layer. In what follows, the working principle of the robot, the design of the adjustable stiffness layer, and its fabrication process is presented. We will characterize the adjustable stiffness layer and estimate the stiffness of the joint in active and inactive states. Also, an algorithm is introduced to find the optimum configuration for joints to reach any point in the work space of the robot.

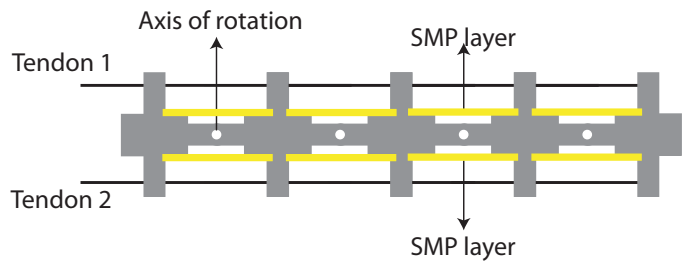

(a)

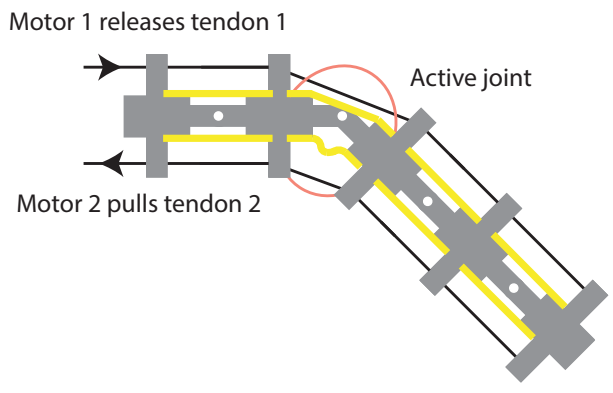

(b)

Fig. 2: The working principle of the under actuated robotic arm. a) Tendons, that are driven by two stepper motors, can actuate each of the 4 folding areas based on their stiffness. b) By activating the second fold while keeping the rest of the folding areas stiff, we are able to direct the actuation to the desired joint.

\section{Under Actuated ARM With AdJustable STIFFNESS JOINTS}

The goal of this research is to maximize the number of DoF and the work space span with minimum number of actuators (one independent input), by introducing a lock mechanism that can activate DoFs on demand. To do this, we use temperature dependent elastic properties of polymers for making lockable joints in an under actuated robotic arm. The material we use is SMP with glass transition temperature of $55{ }^{\circ} \mathrm{C}$ (MM5520 from SMPTechnologies [15]). Above this temperature, the polymer changes its modulus of elasticity by 2 orders of magnitude. This is enough for changing the point of actuation in the robotic arm by selectively locking and unlocking joints. We use shape memory polymer to have a fixed shape for the polymeric layer above its transition temperature and hence more repeatable results. Fig. 1 presents a robotic arm which uses this locking mechanism. In this design, we have four DoFs that can be activated by changing the temperature of the SMP layers. Two stepper motors pull (or release) the tendons that actuate the active joint. In what follows, the working principle of the robot, the fabrication process, and characterization results for the adjustable stiffness elements are presented.

\section{A. Working Principle}

The schematic of the robotic arm depicting its working principle is presented in Fig. 2. Each joint is composed of a revolute joint and two layers of SMP with embedded heaters. We use two stepper motors for actuating different joints. A tendon transmits the actuation to the joints as presented in Fig. 2a. The stiffness of the joints can be adjusted by controlling the temperature of the SMP layers to direct the 


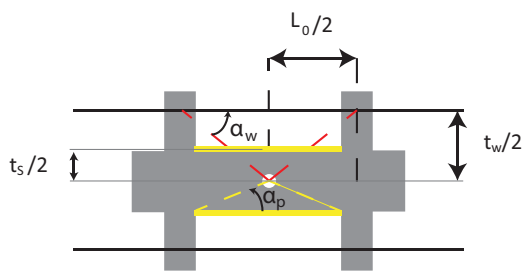

(a)

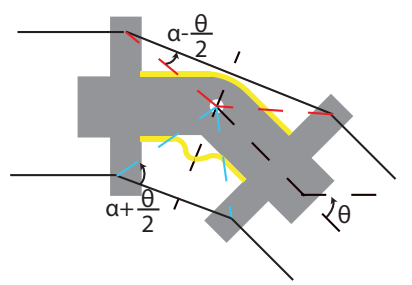

(b)

Fig. 3: Each joint consists of a revolute joint, 2 SMP layers and 2 tendons. (a) shows the joint in its initial state and (b) presents the folded state. In the folded state, the layer in compression collapses (buckles) and the layer in tension stretches.

actuation from the motors to a desired joint as presented in Fig. 2b. Assuming the one points of actuation (stepper motors) as the input and the position of the four joins as the output, the arm presented in Fig. 2 is considered as an under actuated mechanism throughout this paper.

The SMP layers were designed to be thin $(500 \mu \mathrm{m})$ in order to speed up the activation of the folds. To acquire a large enough moment of inertia in the joints, these thin sheets were placed at a distance from the neutral plane. Fig. 3 presents a more detailed schematic of a single joint as it is actuated. In the active fold, the SMP layer in compression would buckle under load and the other layer would stretch. The force in these two layers make the resisting moment in each joint. To evaluate the moment in each joint from the force data for the SMP layers, we have:

$$
M\left(\theta_{0}, \delta \theta, T\right)=F_{t}\left(\theta_{0}, \delta \theta, T\right) \frac{t_{s}}{2}+F_{c}\left(\theta_{0}, \delta \theta, T\right) \frac{t_{s}}{2}
$$

In (1), $M\left(\theta_{0}, \delta \theta, T\right)$ is the moment required for changing the angle of a joint $\delta \theta$ around its initial state $\theta_{0} . F_{t}\left(\theta_{0}, \delta \theta, T\right)$ and $F_{c}\left(\theta_{0}, \delta \theta, T\right)$ are the forces in SMP layers in tension and compression, respectively. All of these are dependent on the initial position of the joint $\left(\theta_{0}\right)$ and the temperature of the SMP layers $(T)$ (in this study, we considered joint properties at room temperature and $80{ }^{\circ} \mathrm{C}$ which are bellow and above the transition temperature, respectively). As presented in Fig. $3 \mathrm{a}, t_{s}$ is the distance between the joint axis and the mid section of SMP layer. Using (1), we can estimate the joint stiffness from the test data on the SMP layers. We chose this approach, instead of directly testing joint's bending stiffness, since such measurements can be used later in design process for making a joint of a certain stiffness. Moreover, this approach provides a better insight on the parameters affecting joint's stiffness. Assuming that the force in the SMP layers is solely a function of their length (governed by the distance between the anchoring points), for the joint stiffness we have:

$$
\begin{aligned}
K_{\text {bend }} & =\frac{\partial M}{\partial \theta} \\
& =\frac{\partial F_{t}}{\partial L_{p t}} \frac{\partial L_{p t}}{\partial \theta} \frac{t_{s}}{2}+\frac{\partial F_{c}}{\partial L_{p c}} \frac{\partial L_{p c}}{\partial \theta} \frac{t_{s}}{2}
\end{aligned}
$$

In (2), $L_{p t}$ and $L_{p c}$ are the length of the polymer layers in tension and compression, respectively. For the layer in tension we have:

$$
L_{p t}=L_{p 0}+\frac{\left(t_{s}\right)}{2} \theta
$$

In (3), $L_{p 0}$ is the initial length of the polymer layer. $L_{p t}$ is different from anchoring point distance since the deformation of the layer is confined by the joint shape as presented in Fig. 3. Differentiating with respect to $\theta$ we have:

$$
\frac{\partial L_{p t}}{\partial \theta}=\frac{\left(t_{s}\right)}{2}
$$

Under compression, the SMP layer buckles and the displacement would cause the buckled arms of the SMP layer to bend. Nonetheless, the force in this layer is a function of the displacement of the two ends that are anchored on the tiles (though this displacement does not translate to in plane stretch and compression of the SMP layer). From Fig. 3 this displacement can be evaluated as:

$$
L_{p c}=L_{p 0} \frac{\cos \left(\alpha_{p}+\theta / 2\right)}{\cos \left(\alpha_{p}\right)}
$$

$\alpha_{p}$ is dipicted in Fig. 3. Differentiating with respect to $\theta$ we have:

$$
\frac{\partial L_{p c}}{\partial \theta}=-L_{p 0} \frac{\sin \left(\alpha_{p}+\theta / 2\right)}{2 \cos \left(\alpha_{p}\right)}
$$

We use (2), (4), (6), and characterization results to estimate the stiffness of the joints. Another relationship we need is the length of the two tendons as a function of the bending angle. As mentioned, we have two stepper motors for actuating the robot but at each moment the tendon that is pulled is what drives the arm and the other motor turns with a speed dependent on the speed of the first motor to provide the excess length needed for the second tendon. Based on Fig. 3b, for the tendon on the compression side of the arm (the driving tendon), we have:

$$
L_{w c}=L_{w 0} \frac{\cos \left(\alpha_{w}+\theta / 2\right)}{\cos \left(\alpha_{w}\right)}
$$

And for the tendon on the tension side (the follower tendon), we have:

$$
L_{w t}=L_{w 0} \frac{\cos \left(\alpha_{w}-\theta / 2\right)}{\cos \left(\alpha_{w}\right)}
$$

The rotation angle of each motor then is calculated as: 


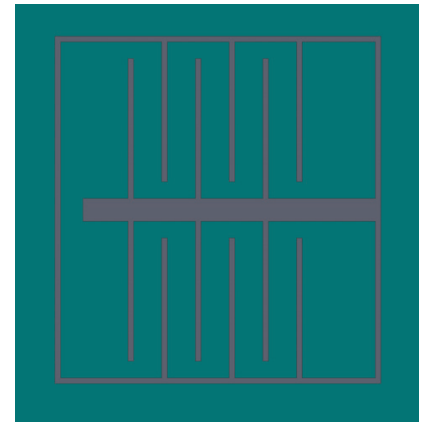

(a)

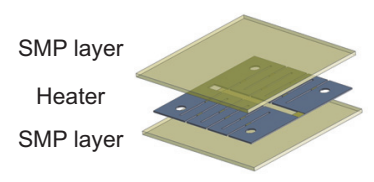

(d)



(b)

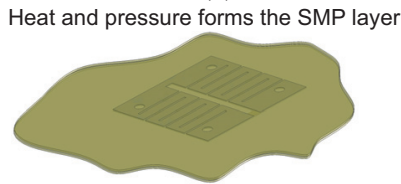

(e)

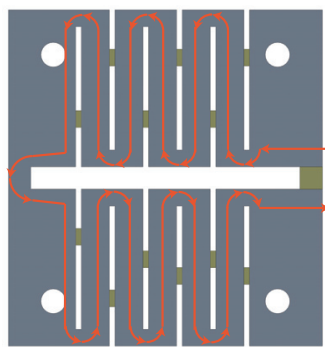

(c)

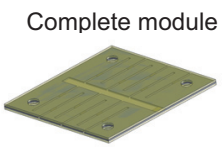

(f)

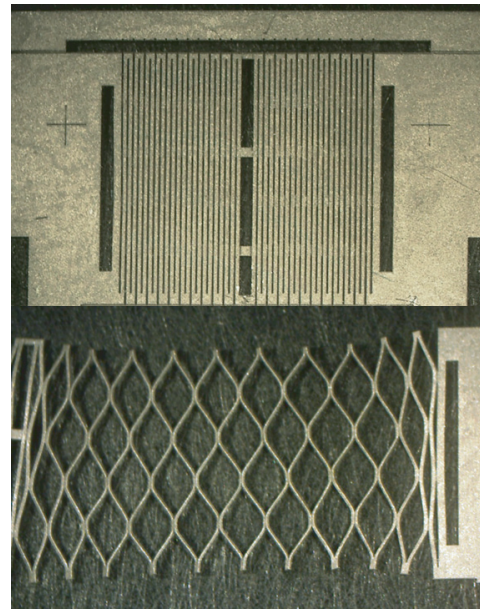

$\underline{2 \mathrm{~mm}}$

$(\mathrm{g})$

Fig. 4: Fabrication process of the SMP layer with embedded heater. For clarity, the dimensions in the schematics are not to scale. (a) A serpentine path, which makes the heating element, is cut through the etchant resist layer. (b) The mesh structure, which makes the heater stretchable and the outline of the heater is cut through all layers. (c) The Inconel in the exposed areas is etched and the etchant resist is cleaned. The red line shows the conductive path of the heater. (d) The heater is sandwiched between 2 SMP layers. (e) High temperature and pressure form the SMP layers around the heater. (f) The outline of the module is cut and its ready for assembly. (g) The fabricated heater in its initial and stretched states.

$$
\Theta_{\text {motor }}=\frac{\Delta L}{R_{\text {shaft }}}
$$

In which $\Delta L$ is the tendon length needed to be pulled or released by each motor and $R_{\text {shaft }}$ is the diameter of the shaft around which the tendon is turned.

\section{B. Design and Fabrication of Arm with Adjustable Stiffness Joints}

The main body of the robotic arm is 3D printed and assembled with the SMP layers and stepper motors to make the device presented in Fig. 1. The stepper motors have 64 steps per revolution. With 64 times reduction in its gear system, it provides accurate enough position control. The main component in the present design is the adjustable stiffness SMP layer with embedded heater. Because of the low thermal conductivity of the polymer, we need to have the heaters embedded inside the adjustable stiffness layer. So we need a heater that can endure repeatable large deformations without loss of functionality. To make the heating elements, we used Inconel Polyimide laminate $(20 \mu \mathrm{m}$ Inconel and $50 \mu \mathrm{m}$ Polyimide). First, we cover the surface of the metal with etchant resist. Then, a serpentine pattern that makes a long path (to increase resistance) is cut on the Inconel and the resist without cutting through the Kapton layer (Fig. 4a). By etching the metal in the exposed parts at this stage we would get the unstretchable heaters introduced previously in [7]. Here, we cut a mesh structure, which makes the heater stretchable through all layers, along the serpentine path. Fig. $4 \mathrm{~b}$ presents the heater at the end of this process and after cutting its outline. Next, Inconel along the serpentine path is etched away in etching tank and the etchant resist is cleaned in an acetone bath. Since the power required for cutting the metal layer is much higher than what is required for the Polyimide layer, processing the heater by burning away the metal without cutting through Polyimide is not possible and using the wet etching process is necessary to make the serpentine path. Fig. 4c presents the heater in its final form and the electric current path. Fig. $4 \mathrm{~g}$ presents the fabricated heater in its initial state and when it is stretched to more that twice its initial size without loosing conductivity. There are some additional details in the design which are omitted in the schematics to avoid confusion. One is the gaps at the two end of the heater and in the middle (Fig. 4g). We have added these gaps to the design to ensure that the embedded heater would move with the SMP layer and prevent delamination. Another detail is the outer frame of the heater in its initial state. This frame is designed to prevent deformation during the integration process which is explained shortly. After integration, the outer frame is cut and the module will be stretchable.

After preparing the heater, wires are connected to its terminals and it is pressed between 2 layers of SMP (each $500 \pm 50 \mu \mathrm{m}$ thick) in a heat press at $160{ }^{\circ} \mathrm{C}$ (Fig. 4e) for 15 minutes. We used two spacers, each $500 \mu \mathrm{m}$ thick, to control the overall thickness of the heater embedded SMP layer. As mentioned, the solid frame of the heater is designed to keep it from deformation under pressure in this step. After integration, the outline and the mounting holes are cut (Fig. 4f) and the SMP layer is ready for assembly.

\section{Adjustable Stiffness Module Characterization}

Due to the residual strain, the deformation of the SMP layer in the robotic arm is more complicated than simple elongation and contraction (it is a combination of buckling and bending). Because of this, we first need to characterize the adjustable stiffness layer to be able to estimate the stiffness of the joints (using the material properties to estimate 
the stiffness would be rather complicated). In this research, we characterized the adjustable stiffness modules in two states: first, at room temperature and second, at $80{ }^{\circ} \mathrm{C}$. The element was heated by passing current through the heaters embedded in the adjustable stiffness modules (Fig. 4f). We characterized the elastic behavior of the adjustable stiffness layer in tension $\left(\partial F_{t} / \partial L_{p t}\right)$ and compression $\left(\partial F_{c} / \partial L_{p c}\right)$. Later, using this result and (2), we evaluate the joint stiffness. To characterize the linear stiffness of the heater embedded SMP layer, we used the same geometry as would be used in the arm. The tests were carried out in a C42 universal testing system from MTS. As mentioned, the stiffness of the layer is a functions of the the initial shape and temperature. In the characterization tests, the sample was heated by passing current through the embedded heater. The temperature is monitored by a thermal camera (FLIR A35) and the heating power was set to achieve the mean temperature of 80 ${ }^{\circ} \mathrm{C}$ on the SMP layer to ensure that it has surpassed the glass transition temperature. To characterize the stiffness in different initial deformations, we deformed the SMP layer while it was in rubbery state (high temperature) to a given initial displacement and performed the tensile tests around the initial displacement in glassy and rubbery states. Fig. 5 presents the results of this test.

In the region where the SMP layer is in the buckled state at its initial deformation, the stiffness of the layer both in compression and tension is lower compared to the points where SMP layer is straight. In points with initial buckled shape, the displacement causes bending in the SMP layer which requires much less energy compared to stretching or compressing the layer in its plane. This explains the higher stiffness in positive initial displacements in Fig. 5 where the SMP layer is straight. Due to the shape memory effect, after each stretch, the SMP layer recovers the deformation when it goes back to no displacement point at rubbery state. But some residual deformations will remain which makes the initial buckled shape in the layer not only in negative displacements but also at zero and some positive values. Most of the unrecoverable deformation happens in the first cycle

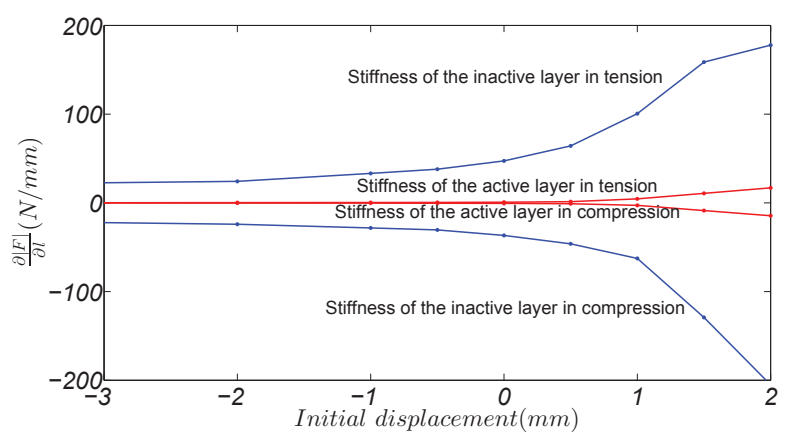

Fig. 5: Linear stiffness of SMP layer in different initial deformations. This plot presents the stiffness of the SMP module in tension and compression in inactive and active states. The stiffness changes by order of magnitude when the polymer enters the rubbery state. Using this, we can activate joints in the under actuated arm on demand. The stiffness in compression is presented with negative sign for better illustration.



Fig. 6: Joint stiffness in active and inactive states as a function of the bending angle. This is estimated from the linear stiffness test results. Using the stiffness change between rubbery and glass states in polymers, we can activate joints on demand.

of loading. In Fig. 5, the test results for a sample after 10 cycles of loading, when the layer has reached a rather stable state, is presented.

Using these test results and according to (2), joint's stiffness is estimated and presented for different bending angles in Fig. 6. The considerable stiffness change in the whole range of motion between enabled and disabled states, presented in this figure, makes it possible to disable different joints in the robotic arm on demand. It would be possible to get more uniform stiffness in the joints by overloading the SMP layers in a higher displacement compared to the normal working range in the initial cycles. This results in larger residual deformation in the SMP layer and gives the buckled shape to the SMP layer throughout its working range. In this way, we will remain in the rather flat range of the stiffness graphs in Fig. 5). This and other methods for forming the SMP layer to get more uniform joint stiffness will be studied in future.

\section{CONFIGURATION PLANNER}

In the four DoF arm introduced, we can have more than one configuration to reach a desired goal position. Among these, the most time efficient is the one that uses the minimum number of joints, considering that the activation/deactivation of the joints is the most time consuming part of the process. Finding this configuration requires the solution of two complex problems.

1) Determining the active joints.

2) Solving the inverse kinematic by considering only the predefined active joints.

To determine the active joints, we need to model robot's reachable space $\left(R_{D} \in \mathbb{R}^{d}\right)$ and its joint configuration

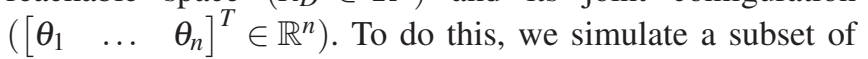
the possible motions of the robot by testing systematically the displacements of its joints. For the under actuated robot, we sample uniformly ${ }^{1}$ its displacement, which yields $18^{4}$ feasible tip positions. The number of the extended workspace models is $\mathscr{N}=\sum_{i=0}^{n-1}\left(\begin{array}{c}n \\ i\end{array}\right)$; where the extended configurations

${ }^{1}$ Eighteen slices for each joint 
Algorithm 1 Finding the minimum active joints for reaching the desired tip position

\section{Offline}

Learning phase: Constructing the extended workspace models

$P_{0}\left(\eta \mid \pi^{0}, \mu^{0}, \Sigma^{0}\right)$, Training data set $=T_{0}$

For i: 1 to $\mathscr{N}$ End

$P_{i}\left(\eta \mid \pi^{i}, \mu^{i}, \Sigma^{i}\right)$, Training data set $=T_{i}$

\section{Online}

Planning phase: finding the minimum active joints

Solution $=$ False

$\Theta=\emptyset$

For i: 1 to $\mathscr{N}$

If $P_{0}\left(R_{D} \mid \pi^{0}, \mu^{0}, \Sigma^{0}\right)>P_{\min }^{0}$

For i: 1 to $\mathscr{N}$

If $P_{i}\left(R_{B}^{i} \mid \pi^{i}, \mu^{i}, \Sigma^{i}\right)>P_{\min }^{i}$ and Solution $==$ False

Solution $=$ True

End

The inactive joints are $\Theta^{i}$

End

ELSE

The desired point $R_{D}$ is not in the workspace of robot. End End

corresponding to each model are defined as follows:

$$
\begin{aligned}
& \eta_{i}=\left[\begin{array}{ll}
R_{D} & \Theta_{i}
\end{array}\right]^{T} \quad \forall i \in\{0,1, \ldots, \mathscr{N}\} \\
& \Theta_{0}=[]^{T} \\
& \Theta_{1}=\left[\theta_{1}\right]^{T} \quad \ldots \Theta_{n}=\left[\theta_{n}\right]^{T} \\
& \Theta_{n+1}=\left[\begin{array}{ll}
\theta_{1} & \theta_{2}
\end{array}\right]^{T} \quad \ldots \\
& \Theta_{\mathscr{N}}=\left[\begin{array}{lll}
\theta_{1} & \ldots & \theta_{\mathscr{N}}
\end{array}\right]^{T}
\end{aligned}
$$

We model the density distribution of the tip positions and the joint configurations using GMM models $\left(P_{i}\right)$ [19]. ${ }^{2}$ The trained models $P_{i}$ is defined as follows:

$$
P_{i}\left(\eta_{i} \mid \pi^{i}, \mu^{i}, \Sigma^{i}\right)=\sum_{i=1}^{K_{i}} \pi^{i} N\left(\eta_{i} \mid \mu^{i}, \Sigma^{i}\right)
$$

In fact $P_{0}$ is the model of the reachable space for arm's tip when all the joints are active and $P_{i} \forall i \in\{1, \ldots, \mathscr{N}\}$ are the representative of the reachable space when $\Theta_{i}$ are inactive and fixed to the given configuration. In (12), $\pi^{i}, \mu^{i}, \Sigma^{i}$ are the parameters of $i^{t h}$ Gaussian and correspond to the prior, mean, and covariance matrix, respectively. $N\left(\eta \mid \mu^{i}, \Sigma^{i}\right)$ is normal distribution. Expectation maximization (EM) [20] algorithm is used for calculating these parameters. If $P_{i}\left(\eta_{i} \mid \pi^{i}, \mu^{i}, \Sigma^{i}\right)$ exceeds a reachable space threshold $P_{\text {min }}^{i}$, a given desired point $R_{D}$ is in the reachable space of the robot when $\Theta_{i}$ are locked at the given configurations. $P_{\text {min }}^{i}$ is set at the one standard deviation from the expectation of the training data points in (12). Algorithm 1 summarizes the complete procedure for determining the optimal number of joints for reaching a desired point.

\footnotetext{
${ }^{2}$ The number of Gaussian are determined using BIC criterion. For example, the reachable space model $\left(P_{0}\left(\eta \mid \pi^{0}, \mu^{0}, \Sigma^{0}\right)\right)$ consists of 31 Gaussians.
}

Algorithm 2 Solving the inverse kinematic by using the active joint

$$
\begin{aligned}
& \text { Initialization Construct the Jacobian matrix: } \\
& \text { For } i: 1 \text { to } \mathrm{n} \\
& \text { If } i^{\text {th }} \text { joint is active } \\
& \quad J_{i}=J[i,:] \in \mathbb{R}^{d} \\
& \text { Else } \\
& \quad J_{i}=[0] \in \mathbb{R}^{d} \\
& \text { End } \\
& \text { End } \\
& J_{\text {Disired }}=\left[\begin{array}{lll}
J_{1} & \ldots & J_{n}
\end{array}\right] \\
& J^{+}=J_{\text {Desired }}^{T}\left(J_{\text {Desired }} J_{\text {Desired }}^{T}\right)^{-1}
\end{aligned}
$$

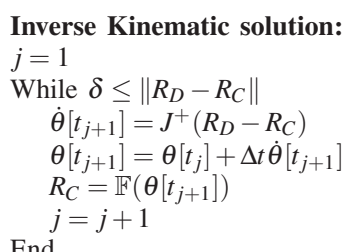

In the second step, inverse kinematic of the arm with just the active joints is solved to determine the proper configuration as presented in Algorithm 2. In brief, in this algorithm, the first-order inverse differential kinematics is used for solving inverse kinematic [21]. The redundancy resolution is solved at the velocity level. In Algorithm 2, $J \in \mathbb{R}^{d \times n}$ is the Jacobian matrix and $J[i,:]$ means the $i^{t h}$ column of the Jacobian matrix. $\Delta t$ is the time step and $\delta$ is a small positive number. $\mathbb{F}: \mathbb{R}^{n} \rightarrow \mathbb{R}^{d}$ is a known forward kinematic function for the robot. The plus + notation is used to define its generalized Moore-Penrose pseudoinverse.

\section{EXPERIMENTAL RESULTS AND DISCUSSION}

In the previous sections, the design of the under-actuated arm with adjustable stiffness joints and an algorithm to find a configuration to reach different points in its work space was introduced. In this section, we report the result of the experiments on this under-actuated arm. As the first test, we chose a configuration of the robot with three joints at the defined joint limit $\left(45^{\circ}\right)$. The SMP components in the joints were successfully tested for deformation up to $90^{\circ}$. The limitation on the joint angle is imposed by the design of the joint and the driving tendon which in larger bending angles gets trapped in the material around the rotation axis. This can easily be solved in the future by changing $t_{w}$ in Fig. 3 to expand the work space. Fig. 7 presents the actuation sequence of the arm to reach the first test point. In each step, the SMP modules of the active joint are heated to make that joint complaint. Fifteen seconds was allocated for deactivation of the previously active joint and heating the SMP elements of the new active joint. The error in arm's tip positioning is mainly due to the tolerances forced by the 3D printer, errors in assembly, and the small deformation of the joints which are locked. The accuracy can be improved by revising the design but eventually feedback from the joint angles is necessary to get more repeatable and accurate motion.

The second test was designed to reach a point in the work 


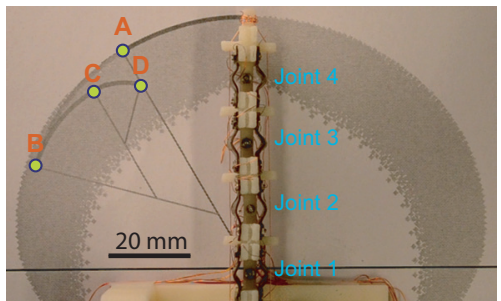

(a)



(b)

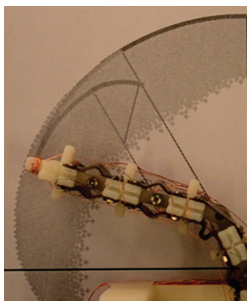

(c)



(d)

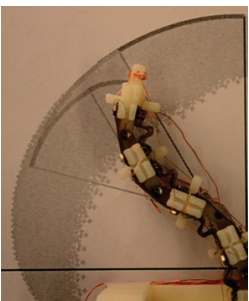

(e)

Fig. 7: Position control of the under actuated arm. The shadowed area in the background shows the work space of the arm (considering $45^{\circ}$ joint limit). a) The goal position is [-26 44] mm which is accessible by moving all four joints with the angles [30 45 -45 -45]. A, B, C, and D present the desired tip position at the end of each step. (b), (c), (d), (e) Shows first, second, third and forth joints reaching their desired orientations. (Refer to the 1st desired point video in supplementary data)

space which is reachable with different configurations ([$2151] \mathrm{mm})$. The planner suggests that it is reachable with motion of only 2 joints (proposed joint configuration is [0 0 35 19]). As presented in Fig. 8, other configurations for the arm using more DoFs are also possible for reaching the same position (as an example [-10 43 -19 39] is a configuration that reaches the same point using all 4 joints). But by choosing the configuration that requires the minimum number of active joint, the algorithm presented in Section 3 suggests a more time efficient configuration.

\section{CONCLUSION AND FUTURE WORK}

We showed a practical application for modulus of elasticity change in SMP around its glass transition temperature for controlling the stiffness of joints in an under-actuated mechanism. The usage of SMP in meso-scale robotic applications faces challenges such as embedding heating component in the robot. For this prototype, we proposed a design based on a thin SMP layer with an embedded metallic heater as the adjustable stiffness component. This integration of the microheater and SMP layer confirmed 16-150 times decrease in the joint stiffness over the glass transition temperature of the polymer. As pointed out, this ratio highly depends on the initial displacement of the layer. This change of material property is well utilized to lock three joints in a four DoF arm to direct the input from stepper motors to the only active joint at each moment. To find the optimum configuration to reach different goal positions, we trained Gaussian mixture models for different cases where different combinations of

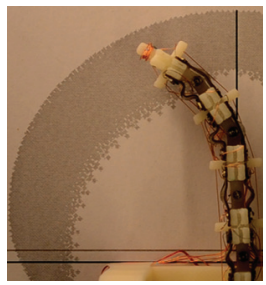

(a)



(b)
Fig. 8: Two trajectories and configurations for the arm to reach the same goal position[-21 51 l $\mathrm{mm}$. The joint angles are (a) [ [ $\left.\begin{array}{llll}0 & 0 & 35 & 19\end{array}\right]$ for the case where only the third and the fourth joints are active and (b) [ $\left[\begin{array}{lll}-10 & 43 & -19\end{array}\right.$ 39] when all joints are active. (Refer to the 2 nd desired point video in supplementary data) joints were used. Then, for any given goal position, using the probability of its presence in each model, the planner decides on how many joints it would need and by solving the inverse kinematics problem for those joints, finds the desired configuration. We have demonstrated position control of all the joints and the tip of the device, using the proposed joint locking mechanism.

Despite the discrepancies in the prototype and assembly, the open-loop control showed promising results. The immediate future step for the adjustable stiffness joints are to get direct feedback from joint angles for more accurate position control. We will also revisit the joint design to have more uniform stiffness throughout the range of motion. Another topic for future studies is the possibility of stiffness control in increments to activate more than one joint to different degrees at the same time.

\section{ACKNOWLEDGMENT}

This work was supported by Swiss National Center for Competence in Research in robotics and EU project AlterEgo (grant \#600610). The authors would like to thank Antoine Foba Amon Jr. and Adriana Basbous Moukarzel for their help in refining the fabrication process.

\section{REFERENCES}

[1] A. Degani, H. Choset, A. Wolf, and M. A. Zenati, "Highly articulated robotic probe for minimally invasive surgery," in Robotics and Automation, 2006. ICRA 2006. Proceedings 2006 IEEE International Conference on, pp. 4167-4172.

[2] Y. Haga, Y. Tanahashi, and M. Esashi, "Small diameter active catheter using shape memory alloy," in Micro Electro Mechanical Systems, 1998. MEMS 98. Proceedings., The Eleventh Annual International Workshop on, pp. 419-424.

[3] R. Buckingham and A. Graham, "Snake-arm robotsa new tool for the aerospace industry," SAE Technical Paper, Tech. Rep., 2003.

[4] M. Grebenstein, S. Albu, x, A. ffer, T. Bahls, M. Chalon, O. Eiberger, W. Friedl, R. Gruber, S. Haddadin, U. Hagn, R. Haslinger, H. Hoppner, S. Jorg, M. Nickl, A. Nothhelfer, F. Petit, J. Reill, N. Seitz, T. Wimbock, S. Wolf, T. Wusthoff, and G. Hirzinger, "The dlr hand arm system," in Robotics and Automation (ICRA), 2011 IEEE International Conference on, pp. 3175-3182.

[5] A. Kochan, "Shadow delivers first hand," Industrial Robot: An International Journal, vol. 32, no. 1, pp. 15-16, 2005.

[6] A. Firouzeh, M. Ozmaeian, A. Alasty, and A. Iraji zad, "An ipmc-made deformable-ring-like robot," Smart Materials and Structures, vol. 21, no. 6, p. 065011, 2012.

[7] A. Firouzeh, S. Yi, L. Hyunchul, and J. Paik, "Sensor and actuator integrated low-profile robotic origami," in Intelligent Robots and Systems (IROS), 2013 IEEE/RSJ International Conference on, pp. 4937-4944. 
[8] L. Zheng and D. Ruxu, "Expanding workspace of underactuated flexible manipulators by actively deploying constraints," in Robotics and Automation (ICRA), 2014 IEEE International Conference on, pp. 2901-2906.

[9] A. Taherifar, A. Alasty, H. Salarieh, and M. Boroushaki, "Path planning for a hyper-redundant manipulator with lockable joints using pso," in Robotics and Mechatronics (ICRoM), 2013 First RSI/ISM International Conference on, pp. 224-229.

[10] N. G. Cheng, M. B. Lobovsky, S. J. Keating, A. M. Setapen, K. I. Gero, A. E. Hosoi, and K. D. Iagnemma, "Design and analysis of a robust, low-cost, highly articulated manipulator enabled by jamming of granular media," in Robotics and Automation (ICRA), 2012 IEEE International Conference on, pp. 4328-4333.

[11] M. Cianchetti, T. Ranzani, G. Gerboni, I. De Falco, C. Laschi, and A. Menciassi, "Stiff-flop surgical manipulator: Mechanical design and experimental characterization of the single module," in Intelligent Robots and Systems (IROS), 2013 IEEE/RSJ International Conference on, pp. 3576-3581.

[12] S. Wanliang, L. Tong, and M. Carmel, "Soft-matter composites with electrically tunable elastic rigidity," Smart Materials and Structures, vol. 22 , no. 8 , p. $085005,2013$.

[13] N. G. Cheng, A. Gopinath, L. Wang, K. Iagnemma, and A. E. Hosoi, "Thermally tunable, self-healing composites for soft robotic applications," Macromolecular Materials and Engineering, pp. n/an/a, 2014.

[14] L. Hines, V. Arabagi, and M. Sitti, "Shape memory polymer-based flexure stiffness control in a miniature flapping-wing robot," Robotics, IEEE Transactions on, vol. 28, no. 4, pp. 987-990, 2012.

[15] http://www2.smptechno.com/en/smp/, "Shape memory polymer properties."

[16] Y. Zhang, S. Ge, and T. Lee, "A unified quadratic-programming-based dynamical system approach to joint torque optimization of physically constrained redundant manipulators," IEEE Transactions on Systems, Man, and Cybernetics, Part B: Cybernetics, vol. 34, no. 5, pp. 21262132, 2004, cited By (since 1996)96.

[17] A. D. Marchese, R. K. Katzschmann, and D. Rus, "Whole arm planning for a soft and highly compliant 2d robotic manipulator," 2014.

[18] M. Shimizu, H. Kakuya, W.-K. Yoon, K. Kitagaki, and K. Kosuge, "Analytical inverse kinematic computation for 7-dof redundant manipulators with joint limits and its application to redundancy resolution," IEEE Transactions on Robotics, vol. 24, no. 5, pp. 1131-1142, 2008, cited By (since 1996) 37.

[19] S. Kim, A. Shukla, and A. Billard, "Catching objects in flight," IEEE Transactions on Robotics, 2014.

[20] C. M. Bishop, Pattern Recognition and Machine Learning (Information Science and Statistics). Secaucus, NJ, USA: Springer-Verlag New York, Inc., 2006.

[21] S. Chiaverini, G. Oriolo, and I. Walker, "Kinematically redundant manipulators," in Springer Handbook of Robotics, B. Siciliano and O. Khatib, Eds. Springer Berlin Heidelberg, 2008, pp. 245-268. 